\title{
EVALUATION OF PHYSICO-CHEMICAL COMPOSITION IN BULBS OF RED, YELLOW, AND WHITE ONION (ALLIUM CEPA L.) GENOTYPES OF SUB-TROPICAL INDIA
}

\author{
I. Kaur*, N. Chawla, A.S. Dhatt and M. Kaur \\ Punjab Agricultural University, Ludhiana, 141001-India
}

(Received: 24 April 2020; accepted: 17 July 2020)

\begin{abstract}
In this study, thirty onion (Allium cepa L.) genotypes grown in sub-tropical region of India were analysed for different physico-chemical attributes. There were significant differences among genotypes, and the onion genotypes showed a tendency to be classified according to different colours. The cultivars of the same colour exhibited similar tendencies in terms of accumulating most of the analysed components. About 1.78 fold variation in dry matter (\%) and 2 fold variation in fresh weight per bulb were recorded among coloured onions. Red genotype D-888-B possessed maximum contents of TS and NRS, while the yellow coloured genotype POH-5 accumulated highest RS and lowest NRS contents. Maximum values of fructans (3.68 g/100 g DW), AIS (6 g/100 g DW), protein (10.61 $\mathrm{g} / 100 \mathrm{~g} \mathrm{DW}$ ), and FAA (4.24 g/100 g DW) were also found in red coloured genotypes D-715-B, D-97-B, PR-305, and D-PS-121-B, respectively. Proline content in different genotypes was found to vary about 6.9 fold. The correlation studies showed a positive relationship between most of the quality parameters. Our results suggested that red group genotypes were better than yellow and white groups for all the studied parameters except for RS, which makes red genotypes more suitable for processing purposes.
\end{abstract}

Keywords: coloured onions, biochemical parameters, correlation analysis

Onion (Allium cepa L.) is a bulbous vegetable, belonging to family Alliaceae, cultivated in temperate, tropical, and sub-tropical regions throughout the world. India is the second largest onion growing country in the world after China. Indian onions are famous for their pungency, which are consumed both within the country and exported to several countries as well. Onion genotypes are grouped into red, yellow, and white colours based on flavonoid contents (Rodrigues et al., 2017). These different coloured onions have different properties like flavour, taste, degrees of pungency, etc.

Different biochemical attributes contribute variably towards quality of onion bulb in these coloured types. Sugars contribute to organoleptic properties, sweetness, and are responsible for distinctive flavour, aroma, and quality. Fresh weight and dry weight of onion are genotypic characteristics. Onion contains significant amount of protein and is considered a high-energy food (BHATTACHARJEE et al., 2013). Amino acids have important role in protein synthesis as well as flavour and pungency development. Cysteine mainly supplies sulphur for most of the organic sulphur compounds in Allium. All these parameters are important for processing and export quality of bulbs (SIMON, 1995). Onion contributes flavour to food without significantly raising the caloric content. It is also a good source of biologically active antioxidant components like phenolic compounds, flavonoids, sulphur active compounds, and various minerals (ViJn \& SMeEKens, 1999; Platel \& SRinivasan, 2016).

In India, onion cultivation is very important and the growing area is increasing because of favourable climatic and soil conditions, availability of suitable cultivars for different

\footnotetext{
* To whom correspondence should be addressed.

Phone: +91 8968615353,+91 9988500406; e-mails: inderpalkaur06@gmail.com, inderpal-bcm@pau.edu
} 
seasons and their production technology. The crop is grown in two seasons, i.e. Rabi and Kharif. There are few popular cultivars among the farmers in the subtropical regions of the country, but many other genotypes exist in the different colour groups (red, yellow, and white), which have not been characterised yet. Therefore, the present investigations were undertaken to study the physico-chemical composition of thirty onion genotypes ( 21 red, 5 yellow, and 4 white). As onion holds a significant place in Indian diet and markets, this data on different onion genotypes will be useful for consumers, farmers, stakeholders, and vegetable breeders for selecting the promising ones among the red, yellow, and white onion genotypes for varied purposes like internal market, processing, and export.

\section{Materials and methods}

\subsection{Trial location and experimental material}

The experiment was conducted at Vegetable Research Farm, Department of Vegetable Science, PAU, Ludhiana (latitude $30^{\circ} 53^{\prime} \mathrm{N}$, longitude $75^{\circ} 48^{\prime} \mathrm{E}$; elevation $244 \mathrm{~m}$ ), India during the year 2018. Ludhiana features humid sub-tropical climate with average maximum and minimum temperatures of 35.8 and $2.7^{\circ} \mathrm{C}$, respectively, and annual rainfall of $733 \mathrm{~mm}$. Thirty genotypes of onions comprising of 20 red ( D-PS-121-B, D-715-B, D-305-B, D-266-B, 65 B, D-31-B, D-4-10-B, D-888-B, Rec-1404, Rec-1410, PDR-1260, PR-10-853, PR-10367, PRO-6, Punjab Naroya, PDR-821, Rec-1417, PR-305, POH-2, and POH-3), 6 yellow (D-97-B, D-30-B, PBY10-214, POH-1, POH-4, and POH-5), and 4 white (D-73-B, D-48-B, PW-731035, and Punjab White) genotypes were grown. Planting was done at a spacing of 15 $\mathrm{cm}$ between rows and $7.5 \mathrm{~cm}$ between plants. The plants were furrow irrigated at 7-10 days intervals, and watering was stopped a fortnight before bulb harvesting. Farmyard manure (20 tonnes per acre) and fertilisers (containing $40 \mathrm{~kg}$ nitrogen, $20 \mathrm{~kg}$ phosphorus and $20 \mathrm{~kg}$ potassium, per acre) were applied to the plants. Whole farmyard manure, phosphorus, potassium, and half of nitrogen were applied before transplanting, and the remaining dose of nitrogen was applied 4 weeks after transplanting. Onions were harvested after maturity, when leaves dried down. The freshly harvested onions were cured in field and transported to the Biochemistry laboratory of Department of Vegetable Science, Punjab Agricultural University for biochemical analysis.

\subsection{Physical parameters}

For calculating bulb weight, randomly five onions with no visible defect were weighed, and the final weight was calculated by the average of these five in grams. Dry weight (\%) was calculated by drying the blended onion samples in hot air oven at temperature of $60 \pm 5^{\circ} \mathrm{C}$ for 48 hours with proper air circulation in triplicates.

\subsection{Biochemical parameters}

Outer dried scales were removed and coloured onion flesh was cut into pieces, dried, and used to estimate the biochemical parameters. The samples were taken as $100 \mathrm{mg}$ dried powder in triplicates. Total soluble sugars $(\mathrm{g} / 100 \mathrm{~g} \mathrm{DW})$ of dried samples were estimated by the method described by DuBoIs and co-workers (1956). Reducing sugars (g/100 g DW) were determined by method of Somogyi (1952). Alcohol insoluble solids (g/100 g DW) were extracted and estimated by method of MoYer and Holgate (1948). Fructan content (g/100 g 
DW) was estimated by method provided by McRarY and SlatTery (1945). Total soluble protein content $(\mathrm{g} / 100 \mathrm{~g}$ DW) in dried onions was determined by the method given by LowRY and co-workers (1951). Free amino acid content was determined by method described by LEE and TAKahashi (1966). Proline content was estimated as reported by Bates (1973).

\subsection{Statistical analysis}

Results were analysed by the Analysis of Variance using SAS version 9.3 (SAS Institute, Inc, 1992; Cary, NC, USA) with completely randomised design. Mean comparisons were performed by Tukey's test with significance effects $\mathrm{P}<0.05$. Results were presented as mean \pm standard deviation for triplicates. The data was subjected to the Pearson correlation coefficient for the analysis of correlation between parameters.

\section{Results and discussion}

\subsection{Dry weight (DW) and fresh weight (FW)}

The results presented in Table 1A and 1B show that there was a significant difference in the contents of dry matter (\%) and fresh weight per bulb among the 30 onion genotypes. Dry weight ranged from $8.92 \mathrm{~g}$ (D-48-B) to $15.87 \mathrm{~g}$ (Punjab Naroya), while fresh weight ranged from $57.87 \mathrm{~g}$ (D-73-B) to $97.73 \mathrm{~g}$ (PR-10-853). The high level of variability could be due to genetic differences among the cultivars. A significant difference was also observed among the three colour types (Table 2). Highest mean dry weight content and fresh weight per bulb were found in red group onions as $13.01 \mathrm{~g}$ and $72.68 \mathrm{~g}$, respectively, while the lowest were recorded in the white group. The mean dry weight values obtained are similar to those reported in previous studies by JAIME and co-workers (2001) and JuRGIEL-MALECKA and co-workers (2015). Azoom and co-workers (2015) reported significant differences in moisture content and fresh weight among the red and yellow coloured cultivars, having maximum values in red cultivars and minimum in yellow ones.

Table $1 A$ and $1 B$ Physico-chemical parameters in different coloured onion genotypes

\begin{tabular}{|c|c|c|c|c|c|c|}
\hline Colour & Genotype & $\mathrm{DM}$ & FW & TS & $\mathrm{RS}$ & NRS \\
\hline \multirow[t]{12}{*}{ Red } & D-PS-121-B & $11.61 \pm 0.01^{\mathrm{jk}}$ & $72.80 \pm 2.04^{\mathrm{ef}}$ & $47.22 \pm 0.70^{\mathrm{ef}}$ & $15.86 \pm 0.08^{b c}$ & $31.36 \pm 0.66^{\text {defgh }}$ \\
\hline & D-715-B & $13.14 \pm 0.44^{\mathrm{ef}}$ & $48.00 \pm 3.27^{\mathrm{n}}$ & $51.09 \pm 0.64^{\mathrm{c}}$ & $10.17 \pm 0.25^{1}$ & $40.92 \pm 0.62^{b}$ \\
\hline & D-305-B & $10.14 \pm 0.03^{\mathrm{n}}$ & $61.00 \pm 2.01^{\mathrm{kl}}$ & $47.48 \pm 0.49^{\mathrm{de}}$ & $14.11 \pm 0.25^{\mathrm{f}}$ & $33.37 \pm 0.25^{\text {cdef }}$ \\
\hline & D-266-B & $13.61 \pm 0.01^{\mathrm{de}}$ & $80.87 \pm 3.24^{\mathrm{cd}}$ & $42.91 \pm 0.32^{\text {hijk }}$ & $15.09 \pm 0.09^{\mathrm{e}}$ & $27.83 \pm 0.38^{\mathrm{ij}}$ \\
\hline & $65 \mathrm{~B}$ & $10.56 \pm 0.03^{\mathrm{mn}}$ & $69.87 \pm 1.41^{\text {fghij }}$ & $47.22 \pm 0.85^{\mathrm{ef}}$ & $11.33 \pm 0.19^{\mathrm{jk}}$ & $35.89 \pm 0.93^{\mathrm{c}}$ \\
\hline & D-31-B & $14.79 \pm 0.13^{b}$ & $71.33 \pm 0.96^{\mathrm{fg}}$ & $55.52 \pm 0.48^{\mathrm{b}}$ & $15.13 \pm 0.15^{\mathrm{e}}$ & $40.39 \pm 0.62^{b}$ \\
\hline & D-4-10-B & $14.83 \pm 0.05^{\mathrm{b}}$ & $63.07 \pm 0.82^{\text {hijkl }}$ & $42.13 \pm 0.75^{\mathrm{ijk}}$ & $12.50 \pm 0.13^{\mathrm{hi}}$ & $29.63 \pm 0.85^{\mathrm{ghi}}$ \\
\hline & D-888-B & $13.56 \pm 0.1^{\mathrm{de}}$ & $58.73 \pm 1.26^{\mathrm{lm}}$ & $61.65 \pm 0.68^{\mathrm{a}}$ & $14.26 \pm .11^{\mathrm{f}}$ & $47.39 \pm 0.79^{\mathrm{a}}$ \\
\hline & Rec-1404 & $13.48 \pm 0.22^{\mathrm{de}}$ & $70.73 \pm 0.93^{\mathrm{fgh}}$ & $44.00 \pm 1.45^{\text {ghij }}$ & $12.40 \pm 0.1^{\mathrm{hi}}$ & $31.60 \pm 1.4^{\text {defgh }}$ \\
\hline & Rec-1410 & $12.65 \pm 0.08^{\mathrm{fg}}$ & $71.00 \pm 1.02^{\text {fgh }}$ & $50.39 \pm 0.5^{\mathrm{cd}}$ & $10.95 \pm 0.2^{\mathrm{k}}$ & $39.44 \pm 0.59^{\mathrm{b}}$ \\
\hline & PDR-1260 & $11.95 \pm 0.09^{h i j}$ & $80.00 \pm 1.8^{\mathrm{de}}$ & $45.65 \pm 0.7^{\mathrm{efgh}}$ & $15.54 \pm 0.17^{\text {cde }}$ & $30.11 \pm 0.87^{\mathrm{ghi}}$ \\
\hline & PR-10-853 & $14.39 \pm 0.13^{\mathrm{bc}}$ & $97.73 \pm 1.32^{\mathrm{a}}$ & $47.17 \pm 0.37^{\mathrm{ef}}$ & $13.44 \pm 0.1^{\mathrm{g}}$ & $33.74 \pm 0.47^{\text {cde }}$ \\
\hline
\end{tabular}




\begin{tabular}{|c|c|c|c|c|c|c|}
\hline Colour & Genotype & $\mathrm{DM}$ & $\mathrm{FW}$ & TS & RS & NRS \\
\hline & PR-10-367 & $14.42 \pm 0.36^{\mathrm{b}}$ & $90.40 \pm 1.23^{\mathrm{ab}}$ & $41.43 \pm 0.55^{\mathrm{ijk}}$ & $15.70 \pm 0.1^{\text {bcde }}$ & $25.74 \pm 0.54^{\mathrm{jk}}$ \\
\hline & PRO-6 & $11.60 \pm 0.07^{\mathrm{jk}}$ & $79.67 \pm 1.57^{\mathrm{de}}$ & $41.04 \pm 0.59^{\mathrm{jkl}}$ & $16.26 \pm 0.12^{\mathrm{b}}$ & $24.78 \pm 0.70^{\mathrm{jkl}}$ \\
\hline & Pb. Naroya & $15.87 \pm 0.07^{\mathrm{a}}$ & $83.67 \pm 1.25$ & $40.83 \pm 0.77^{\mathrm{kl}}$ & $11.49 \pm 0.07^{\mathrm{jk}}$ & $29.34 \pm 0.70^{\mathrm{hi}}$ \\
\hline & PDR-821 & $11.98 \pm 0.01^{\mathrm{hij}}$ & $85.80 \pm 1.72^{\mathrm{bcd}}$ & $41.87 \pm 0.59^{\mathrm{ijk}}$ & $12.18 \pm 0.11^{\mathrm{i}}$ & $29.69 \pm 0.70^{\text {ghi }}$ \\
\hline & Rec-1417 & $13.90 \pm 0.04^{\mathrm{cd}}$ & $52.40 \pm 1.98^{\mathrm{mn}}$ & $38.04 \pm 0.96^{\mathrm{lm}}$ & $15.38 \pm 0.16^{\text {cde }}$ & $22.67 \pm 0.89^{\mathrm{klm}}$ \\
\hline & PR-305 & $12.16 \pm 0.08^{\mathrm{ghi}}$ & $70.60 \pm 2.24^{\text {fghi }}$ & $44.26 \pm 0.81^{\text {fghi }}$ & $9.96 \pm 0.31^{1}$ & $34.30 \pm 0.92^{\mathrm{cd}}$ \\
\hline & POH-2 & $12.67 \pm 0.04^{\mathrm{fg}}$ & $68.13 \pm 1.64^{\text {fghijk }}$ & $36.17 \pm 0.92^{\mathrm{mno}}$ & $15.82 \pm 0.16^{\mathrm{bcd}}$ & $20.35 \pm 1.07^{\mathrm{mn}}$ \\
\hline & POH-3 & $13.74 \pm 0.11^{\mathrm{d}}$ & $61.80 \pm 1.72^{\mathrm{kl}}$ & $36.91 \pm 0.64^{\mathrm{mn}}$ & $14.24 \pm 0.15^{\mathrm{f}}$ & $22.68 \pm 0.68^{\mathrm{klm}}$ \\
\hline \multirow[t]{6}{*}{ Yellow } & D-30-B & $10.92 \pm 0.02^{\operatorname{lm}}$ & $69.87 \pm 1.55^{\text {fghij }}$ & $43.52 \pm 0.59^{\text {ghijk }}$ & $13.16 \pm 0.18^{\mathrm{g}}$ & $30.36 \pm 0.67^{\text {fghi }}$ \\
\hline & D-97-B & $12.08 \pm 0.04^{\mathrm{hij}}$ & $88.67 \pm 6.59^{\mathrm{bc}}$ & $42.61 \pm 0.43^{\mathrm{hijk}}$ & $9.91 \pm 0.18^{1}$ & $32.70 \pm 0.51^{\text {cdefg }}$ \\
\hline & PBY10-214 & $11.18 \pm 0.08^{\mathrm{kl}}$ & $82.40 \pm 1.23^{\mathrm{bcd}}$ & $46.09 \pm 1.21^{\mathrm{efg}}$ & $15.19 \pm 0.10^{\mathrm{de}}$ & $30.90 \pm 1.16^{\text {efghi }}$ \\
\hline & $\mathrm{POH}-1$ & $11.87 \pm 0.07^{\mathrm{ij}}$ & $63.20 \pm 1.07^{\text {hijkl }}$ & $40.96 \pm 1.26^{\mathrm{jkl}}$ & $13.02 \pm 0.17^{\mathrm{gh}}$ & $27.94 \pm 1.42^{\mathrm{ij}}$ \\
\hline & POH-4 & $10.50 \pm 0.06$ & $61.93 \pm 1.48^{\mathrm{jkl}}$ & $41.43 \pm 0.86^{\mathrm{ijk}}$ & $17.67 \pm 0.19^{\mathrm{a}}$ & $23.76 \pm 0.78^{\mathrm{kl}}$ \\
\hline & POH-5 & $12.42 \pm 0.03^{\mathrm{gh}}$ & $68.67 \pm 0.96^{\text {fghijk }}$ & $37.09 \pm 0.59^{\mathrm{mn}}$ & $17.69 \pm 0.16^{\mathrm{a}}$ & $19.40 \pm 0.71^{\mathrm{n}}$ \\
\hline \multirow[t]{6}{*}{ White } & D-73-B & $9.10 \pm 0.06^{\circ}$ & $57.87 \pm 1.55^{\mathrm{lm}}$ & $30.57 \pm 1.21^{\mathrm{p}}$ & $8.20 \pm 0.17^{\mathrm{m}}$ & $22.37 \pm 1.12^{\operatorname{lmn}}$ \\
\hline & D-48-B & $8.92 \pm 0.01^{\circ}$ & $61.47 \pm 1.88^{\mathrm{kl}}$ & $33.70 \pm 1.12^{\mathrm{op}}$ & $8.15 \pm 0.28^{\mathrm{m}}$ & $25.54 \pm 0.88^{\mathrm{jkl}}$ \\
\hline & PW-731035 & $10.14 \pm 0.07^{\mathrm{n}}$ & $62.67 \pm 1.57^{\mathrm{ijk} \mathrm{l}}$ & $34.13 \pm 0.92^{\text {no }}$ & $8.37 \pm 0.18^{\mathrm{m}}$ & $25.76 \pm 0.81^{\mathrm{jk}}$ \\
\hline & $\mathrm{Pb}$. White & $10.12 \pm 0.08^{n}$ & $63.67 \pm 1.25^{\text {ghij }} \mathrm{kl}$ & $34.26 \pm 0.48^{\text {no }}$ & $11.89 \pm 0.13^{\mathrm{ij}}$ & $22.38 \pm 0.47^{\mathrm{lmn}}$ \\
\hline & MEAN & $12.28 \pm 0.05$ & $70.60 \pm 1.56$ & $42.91 \pm 0.75$ & $13.17 \pm 0.19$ & $29.74 \pm 0.82$ \\
\hline & $\mathrm{CD}(5 \%)$ & 0.51 & 8.06 & 3.13 & 0.66 & 3.21 \\
\hline
\end{tabular}

DM: Dry matter (\%); FW: fresh weight per bulb (g); TS: total soluble sugars (g/100 gm DW); RS: reducing sugars (g/100 gm DW); NRS: non-reducing sugars (g/100 g DW);

Values are mean $\pm \mathrm{SD}$ of triplicates; values with different letters in the same column are significantly different (Pp $<0.05)$.

Table 1B Physico-chemical parameters in different coloured onion genotypes

\begin{tabular}{|c|c|c|c|c|c|c|}
\hline Colour & Genotype & Fructans & AIS & Protein & Free AA & Proline \\
\hline \multirow[t]{13}{*}{ Red } & D-PS-121-B & $2.92 \pm 0.08^{b c}$ & $40.67 \pm 5.35^{\text {cdef }}$ & $9.92 \pm 0.45^{\mathrm{ab}}$ & $4.24 \pm 0.25^{\mathrm{a}}$ & $1.04 \pm 0.02^{1}$ \\
\hline & D-715-B & $3.68 \pm 0.1^{\mathrm{a}}$ & $48.67 \pm 2.87^{\mathrm{abc}}$ & $6.77 \pm 0.44^{\mathrm{g}}$ & $2.38 \pm 0.26^{\text {fghi }}$ & $1.51 \pm 0.02^{\mathrm{ef}}$ \\
\hline & D-305-B & $2.39 \pm 0.07^{\text {hij }}$ & $49.67 \pm 3.40^{\mathrm{abc}}$ & $8.48 \pm 0.22^{\text {cde }}$ & $2.09 \pm .09^{\mathrm{ghi}}$ & $1.49 \pm 0.02^{\mathrm{ef}}$ \\
\hline & D-266-B & $2.40 \pm 0.07^{\text {hij }}$ & $39.0 \pm 4.08^{\text {cdef }}$ & $7.20 \pm 0.35^{\mathrm{fg}}$ & $3.48 \pm 0.09^{b c}$ & $1.34 \pm 0.02^{\mathrm{gh}}$ \\
\hline & $65 \mathrm{~B}$ & $2.58 \pm 0.03^{\mathrm{fghi}}$ & $38.0 \pm 3.74^{\text {cdef }}$ & $7.29 \pm 0.27^{\mathrm{efg}}$ & $3.53 \pm 0.25^{\mathrm{bc}}$ & $2.35 \pm 0.02^{b}$ \\
\hline & D-31-B & $2.97 \pm 0.07^{\mathrm{bc}}$ & $51.67 \pm 4.11^{\mathrm{abc}}$ & $6.68 \pm 0.39^{g}$ & $2.42 \pm 0.13^{\text {fghi }}$ & $2.58 \pm 0.01^{\mathrm{a}}$ \\
\hline & D-4-10-B & $2.61 \pm 0.05^{\mathrm{efgh}}$ & $46.0 \pm 4.08^{\mathrm{abcd}}$ & $7.00 \pm 0.3^{\mathrm{fg}}$ & $2.30 \pm 0.11^{\text {fghi }}$ & $2.20 \pm 0.03^{\mathrm{c}}$ \\
\hline & D-888-B & $3.11 \pm 0.05^{\mathrm{b}}$ & $38.33 \pm 4.11^{\text {cdef }}$ & $6.54 \pm 0.28^{g}$ & $2.33 \pm 0.11^{\text {fghi }}$ & $2.32 \pm 0.02^{b}$ \\
\hline & Rec-1404 & $2.75 \pm 0.13^{\text {cdef }}$ & $51.67 \pm 3.68^{\mathrm{abc}}$ & $10.22 \pm 0.28^{\mathrm{a}}$ & $3.62 \pm 0.15^{\mathrm{ab}}$ & $0.97 \pm 0.03^{1}$ \\
\hline & Rec-1410 & $2.91 \pm 0.05^{\mathrm{bcd}}$ & $44.67 \pm 3.68^{\mathrm{bcd}}$ & $7.64 \pm 0.22^{\text {defg }}$ & $2.32 \pm 0.11^{\text {fghi }}$ & $1.76 \pm 0.03^{\mathrm{d}}$ \\
\hline & PDR-1260 & $2.63 \pm 0.09^{\text {defgh }}$ & $50.0 \pm 4.08^{\mathrm{abc}}$ & $9.62 \pm 0.24^{\mathrm{abc}}$ & $3.19 \pm 0.22^{\mathrm{bcd}}$ & $1.18 \pm 0.02^{\mathrm{k}}$ \\
\hline & PR-10-853 & $2.72 \pm 0.04^{\text {cdefg }}$ & $38.67 \pm 4.11^{\text {cdef }}$ & $10.10 \pm 0.31^{\mathrm{a}}$ & $3.58 \pm 0.19^{\mathrm{ab}}$ & $0.79 \pm 0.01^{\mathrm{m}}$ \\
\hline & PR-10-367 & $2.47 \pm 0.05^{\text {ghij }}$ & $42.33 \pm 2.87^{\text {bcde }}$ & $6.99 \pm 0.22^{\mathrm{fg}}$ & $2.14 \pm 0.13^{\text {ghi }}$ & $1.53 \pm 0.02^{\mathrm{e}}$ \\
\hline
\end{tabular}


KAUR et al.: PHYSICO-CHEMICAL COMPOSITION OF SUB-TROPICAL ONION GENOTYPES

\begin{tabular}{|c|c|c|c|c|c|c|}
\hline Colour & Genotype & Fructans & AIS & Protein & Free AA & Proline \\
\hline & PRO-6 & $2.63 \pm 0.04^{\text {defgh }}$ & $48.33 \pm 3.40^{\mathrm{abc}}$ & $6.77 \pm 0.37^{\mathrm{g}}$ & $2.19 \pm 0.11^{\mathrm{ghi}}$ & $0.65 \pm 0.01^{\circ}$ \\
\hline & $\mathrm{Pb}$. Naroya & $2.64 \pm 0.08^{\text {defgh }}$ & $40.67 \pm 4.92^{\text {cdef }}$ & $6.84 \pm 0.31^{\mathrm{fg}}$ & $2.12 \pm 0.1^{\mathrm{ghi}}$ & $1.29 \pm 0.03^{\mathrm{hi}}$ \\
\hline & PDR-821 & $2.77 \pm 0.12^{\text {cdef }}$ & $26.33 \pm 2.05^{f}$ & $10.36 \pm 0.26^{\mathrm{a}}$ & $3.17 \pm 0.23^{\text {bcde }}$ & $0.67 \pm 0.03^{\mathrm{n}}$ \\
\hline & Rec-1417 & $2.77 \pm 0.08^{\text {cdef }}$ & $27.0 \pm 5.72^{\mathrm{f}}$ & $10.44 \pm 0.26^{\mathrm{a}}$ & $3.49 \pm 0.22^{\mathrm{bc}}$ & $0.76 \pm 0.01^{\mathrm{ml}}$ \\
\hline & PR-305 & $2.32 \pm 0.06^{\mathrm{ij}}$ & $43.67 \pm 4.19^{\mathrm{bcd}}$ & $10.61 \pm 0.48^{\mathrm{a}}$ & $3.27 \pm .02^{\mathrm{bcd}}$ & $1.20 \pm 0.03^{\mathrm{jk}}$ \\
\hline & $\mathrm{POH}-2$ & $2.38 \pm 0.05^{\mathrm{hij}}$ & $27.33 \pm 4.19^{\mathrm{ef}}$ & $6.67 \pm 0.5^{\mathrm{g}}$ & $2.02 \pm 0.13^{\mathrm{i}}$ & $0.43 \pm 0.02^{\mathrm{pc}}$ \\
\hline & $\mathrm{POH}-3$ & $2.28 \pm 0.05^{\mathrm{j}}$ & $32.67 \pm 4.50^{\mathrm{def}}$ & $8.71 \pm 0.29^{\mathrm{bcd}}$ & $2.75 \pm 0.23^{\text {defg }}$ & $1.24 \pm 0.02^{\mathrm{ijk}}$ \\
\hline \multirow[t]{6}{*}{ Yellow } & D-30-B & $2.86 \pm 0.06^{\text {bcde }}$ & $47.33 \pm 3.30^{\mathrm{abcd}}$ & $7.29 \pm 0.31^{\mathrm{efg}}$ & $2.74 \pm 0.16^{\text {defg }}$ & $1.43 \pm 0.03^{\mathrm{fg}}$ \\
\hline & D-97-B & $2.37 \pm 0.04^{\mathrm{hij}}$ & $60.0 \pm 5.35^{\mathrm{a}}$ & $8.04 \pm 0.11^{\mathrm{def}}$ & $2.90 \pm 0.19^{\text {cdef }}$ & $1.73 \pm 0.05^{\mathrm{d}}$ \\
\hline & PBY10-214 & $2.63 \pm 0.08^{\text {defgh }}$ & $42.67 \pm 5.56^{\mathrm{bcd}}$ & $7.08 \pm 0.15^{\mathrm{fg}}$ & $1.85 \pm 0.17^{\mathrm{i}}$ & $1.05 \pm 0.01^{1}$ \\
\hline & POH-1 & $2.31 \pm 0.07^{\mathrm{ij}}$ & $43.33 \pm 2.05^{\mathrm{bcd}}$ & $8.55 \pm 0.37^{\mathrm{cd}}$ & $2.37 \pm 0.15^{\text {fghi }}$ & $0.37 \pm 0.01^{\mathrm{q}}$ \\
\hline & $\mathrm{POH}-4$ & $2.57 \pm 0.04^{\text {fghiefgh }}$ & $43.33 \pm 2.49^{\mathrm{bcd}}$ & $6.90 \pm 0.15^{\mathrm{fg}}$ & $2.50 \pm 0.16^{\text {efghi }}$ & $1.69 \pm 0.03^{\mathrm{d}}$ \\
\hline & $\mathrm{POH}-5$ & $2.27 \pm 0.05^{\mathrm{j}}$ & $41.0 \pm 4.9^{\text {cdef }}$ & $7.22 \pm 0.21^{\mathrm{fg}}$ & $2.70 \pm 0.17^{\mathrm{defgh}}$ & $0.48 \pm 0.03^{p}$ \\
\hline \multirow[t]{6}{*}{ White } & D-73-B & $2.22 \pm 0.07^{\mathrm{j}}$ & $56.67 \pm 4.5^{\mathrm{ab}}$ & $6.77 \pm 0.33^{\mathrm{g}}$ & $2.31 \pm 0.1^{\text {fghi }}$ & $1.22 \pm 0.01^{\mathrm{ijk}}$ \\
\hline & D-48-B & $2.38 \pm 0.08^{\text {hij }}$ & $48.67 \pm 3.30^{\mathrm{abc}}$ & $6.54 \pm 0.3^{\mathrm{g}}$ & $2.06 \pm 0.11^{\mathrm{hi}}$ & $1.18 \pm 0.03^{\mathrm{k}}$ \\
\hline & PW-731035 & $2.20 \pm 0.05^{\mathrm{j}}$ & $47.33 \pm 2.87^{\mathrm{abcd}}$ & $6.86 \pm 0.11^{\mathrm{fg}}$ & $1.97 \pm 0.15^{\mathrm{i}}$ & $1.42 \pm 0.02^{\mathrm{fg}}$ \\
\hline & $\mathrm{Pb}$. White & $2.40 \pm 0.07^{\mathrm{hij}}$ & $26.33 \pm 1.70^{\mathrm{f}}$ & $6.79 \pm 0.39^{g}$ & $2.06 \pm 0.16^{\mathrm{hi}}$ & $1.31 \pm 0.02^{\mathrm{h}}$ \\
\hline & MEAN & $2.60 \pm 0.07$ & $42.73 \pm 3.77$ & $7.90 \pm 0.28$ & $2.67 \pm 0.13$ & $1.30 \pm 0.02$ \\
\hline & $\mathrm{CD}(5 \%)$ & 0.28 & 1.53 & 1.22 & 0.67 & 0.48 \\
\hline
\end{tabular}

Fructans (g/100 g DW); AIS: alcohol insoluble solids (g/100 g DW); protein: total soluble protein (g/100 g DW); free AA: free amino acids ( $\mathrm{g} / 100 \mathrm{~g} \mathrm{DW})$; proline $(\mathrm{g} / 100 \mathrm{~g} \mathrm{DW})$

Values are mean $\pm \mathrm{SD}$ of triplicates; values with different letters in the same column are significantly different (Pp $<0.05)$.

Table 2. Average biochemical contents of red, yellow, and white type onion groups

\begin{tabular}{lcccccccccc}
\hline & DM & FW & TS & RS & NRS & Fructans & AIS & Protein & Free AA & Proline \\
\hline Red & $13.01^{\mathrm{a}}$ & $72.68^{\mathrm{a}}$ & $45.03^{\mathrm{a}}$ & $13.41^{\mathrm{a}}$ & $31.61^{\mathrm{a}}$ & $2.68^{\mathrm{a}}$ & $42.2^{\mathrm{c}}$ & $8.23^{\mathrm{a}}$ & $2.83^{\mathrm{a}}$ & $1.38^{\mathrm{a}}$ \\
Yellow & $11.37^{\mathrm{b}}$ & $69.21^{\mathrm{ab}}$ & $41.82^{\mathrm{a}}$ & $15.35^{\mathrm{b}}$ & $26.47^{\mathrm{b}}$ & $2.53^{\mathrm{ab}}$ & $43.5^{\mathrm{b}}$ & $7.41^{\mathrm{ab}}$ & $2.43^{\mathrm{a}}$ & $1.0^{\mathrm{a}}$ \\
White & $9.57^{\mathrm{c}}$ & $61.42^{\mathrm{a}}$ & $33.16^{\mathrm{b}}$ & $9.15^{\mathrm{c}}$ & $24.01^{\mathrm{a}}$ & $2.30^{\mathrm{b}}$ & $44.8^{\mathrm{a}}$ & $6.74^{\mathrm{b}}$ & $2.10^{\mathrm{b}}$ & $1.28^{\mathrm{a}}$ \\
CD $(5 \%)$ & 0.87 & 7.71 & 3.63 & 1.43 & 4.08 & 0.20 & 0.74 & 0.91 & 0.41 & 0.49 \\
\hline
\end{tabular}

DM: Dry matter (\%); FW: fresh weight per bulb (g); TS: total soluble sugars (g/100 gm DW); RS: reducing sugars (g/100 gm DW); NRS: non-reducing sugars (g/100 g DW); fructans (g/100 g DW); AIS: alcohol insoluble solids $(\mathrm{g} / 100 \mathrm{~g} \mathrm{DW})$; protein: total soluble protein $(\mathrm{g} / 100 \mathrm{~g} \mathrm{DW})$; free AA: free amino acids (g/100 g DW); proline (g/100 g DW)

Values with different letters in the same column are significantly different $(\mathrm{P}<0.05)$.

\subsection{Total soluble sugars (TS), reducing sugars (RS) and non-reducing sugars (NRS) contents}

The total soluble sugars, reducing sugars, and non-reducing sugars contents of 30 onion cultivars fell in the ranges of 30.57 to $61.65 \mathrm{~g} / 100 \mathrm{~g} \mathrm{DW}, 8.15$ to $17.69 \mathrm{~g} / 100 \mathrm{~g} \mathrm{DW}$, and 19.40 to 47.39 , respectively (Table $1 \mathrm{~A}$ ). The maximum and minimum content of TS, RS, and 
NRS was recorded in D-888-B and D-73-B, POH-5 and D-48-B, D-88-B and POH-5, respectively. A significant variability was also observed among the three colour types (Table 2). Highest TS and NRS contents were found in red group onions as $45.03 \mathrm{~g} / 100 \mathrm{~g} \mathrm{DW}$ and $31.61 \mathrm{~g} / 100 \mathrm{~g} \mathrm{DW}$, respectively, while the lowest was observed in white group onions as $33.16 \mathrm{~g} / 100 \mathrm{~g}$ DW and $24.01 \mathrm{~g} / 100 \mathrm{~g} \mathrm{DW}$, respectively. For RS content, the maximum average content $(15.35 \mathrm{~g} / 100 \mathrm{~g} \mathrm{DW})$ was found in yellow group, while the minimum $(9.15$ $\mathrm{g} / 100 \mathrm{~g} \mathrm{DW}$ ) was recorded in white group. In previous studies it was observed that red coloured genotypes possessed higher sugar contents than yellow and white genotypes (Jurgiel-MaleCKa et al., 2015; Azoom et al., 2015; and Armand et al., 2018). Similarly, Dhumal and co-workers (2007) reported that among two red and one white coloured cultivars, the red cultivar (N-2-4-1) had the highest contents of total reducing and nonreducing sugars as $16.1,6.69$ and $9.56 \mathrm{~g} / 100 \mathrm{~g}$, respectively, and white colour genotype (Phule Safed) had the lowest contents.

\subsection{Fructans and alcohol insoluble solids (AIS)}

The data presented in Table 1B reveal that among the 30 genotypes studied, the maximum fructans content was recorded in D-715-B (3.68 g/100 g DW), while the lowest amount was found in PW-731035 (2.20 g/100 g DW). JAIME and co-workers (2001) and SuzuKi and CutCLIFEe (1989) reported higher contents of fructans than those found in our studies. There was a significant difference among the studied genotypes with regard to contents of AIS, the highest detected in D-97-B (60.0 g/100 g DW) and the lowest in both PDR-821 and Punjab White (26.33 g/100 g). KAUR and co-workers (2019) found that AIS content was in the range of $226.67-740.00 \mathrm{mg} \mathrm{g}^{-1}$ DW during storage of onion.

As far as the contents of fructans and AIS among different colour groups of onion are concerned, the mean maximum content $(2.68 \mathrm{~g} / 100 \mathrm{~g} \mathrm{DW})$ of fructans was recorded in red group onions, while the mean minimum $(2.30 \mathrm{~g} / 100 \mathrm{~g} \mathrm{DW})$ was recorded in white type onions. On the contrary, the average content of alcohol insoluble solids was found to be maximum (44.8 g/100 g DW) in white group onions, while the minimum (42.2 g/100 g DW) was recorded in red type onions (Table 2).

\subsection{Total soluble protein content, free amino acids (FAA) content, and proline content}

In this study, total soluble protein, free amino acids, and proline contents ranged from 6.54 (D-888-B) to 10.61 (PR-305) g/100 g, 1.85 (PBY10-214) to 4.24 (D-PS-121-B) g/100 g, and 0.37 (POH-1) to 2.58 (D-31-B) g/100 g DW, respectively (Table 1B). The results pertaining to total soluble protein content are in agreement with the previous studies that reported maximum total soluble proteins content in red coloured cultivars of onion followed by yellow coloured cultivars (Azoom et al., 2015). ARMAND and co-workers (2018) reported protein content ranging 9.84-13.4 g/100 g DW in the studied onion cultivars. KUON and BERNHARD (1963) also found very little variation for free amino acid content in between the cultivars with content ranging from 4.7 to $5.6 \mathrm{~g} / 100 \mathrm{~g}$. Similar range of free amino acids in the yellow and white onion cultivars was also reported by INSANI and co-workers (2016).

The average total soluble protein content among three colour types was found to be maximum in red type onions $(8.23 \mathrm{~g} / 100 \mathrm{~g})$, while the minimum $(6.74 \mathrm{~g} / 100 \mathrm{~g})$ was recorded in white type onions (Table 2). Similar trend was seen for the average contents of free amino acids and proline among different colour groups, where the mean maximum amounts of free amino acids and proline were found in red group $(2.83 \mathrm{~g} / 100 \mathrm{~g}$ and $1.38 \mathrm{~g} / 100 \mathrm{~g}$, respectively), 
while the mean minimum was recorded in white and yellow group onions $(2.10$ and $1.0 \mathrm{~g} / 100 \mathrm{~g}$, respectively). In earlier studies carried out under abiotic stress conditions versus non-stressed growing conditions, proline content was found to be in the range 3.57 to $7.63 \mu \mathrm{mol} \mathrm{g}^{-1} \mathrm{FW}$ in non-stressed plants (HANCI \& CEBECI, 2015), which is similar to results reported in our study.

\subsection{Correlation coefficient between biochemical parameters}

Table 3 shows significant maximum positive correlation $(\mathrm{r}=0.917)$ amongst all quality parameters recorded for TS and NRS, indicating a direct relationship between these parameters. Similarly, positive correlation between NRS and fructans $(\mathrm{r}=0.657)$, proline $(\mathrm{r}=0.516)$, and AIS $(\mathrm{r}=0.241)$ was recorded. For protein and free amino acids contents significant positive correlation at $\mathrm{P}<0.01 \quad(\mathrm{r}=0.703)$ was observed. The proportion of high molecular weight compounds increased as dry matter increased, as evidenced by linear positive relationship between DW and TS $(\mathrm{r}=0.378)$, between DW and RS $(\mathrm{r}=0.306)$, and between DW and NRS $(r=0.253)$, while a lesser relationship was observed between dry matter and protein, proline, and free amino acids. JAIME and co-workers (2001) reported strong positive correlation between fructans and sugars of studied onion cultivars.

Table 3. Pearson correlation coefficients between different parameters studied for thirty onion genotypes

\begin{tabular}{|c|c|c|c|c|c|c|c|c|c|}
\hline & $\mathrm{DM}$ & FW & TS & RS & NRS & AIS & Fructans & Protein & Free AA \\
\hline FW & 0.286 & 1 & & & & & & & \\
\hline TS & $0.378^{*}$ & 0.095 & 1 & & & & & & \\
\hline RS & 0.306 & 0.176 & 0.209 & 1 & & & & & \\
\hline NRS & 0.253 & 0.024 & $0.917 * *$ & -0.198 & 1 & & & & \\
\hline AIS & -0.171 & 0.037 & 0.124 & -0.287 & 0.241 & 1 & & & \\
\hline Fructans & 0.337 & -0.125 & $0.687 * *$ & 0.077 & $0.657 * *$ & 0.015 & 1 & & \\
\hline Protein & 0.143 & 0.209 & 0.062 & 0.055 & 0.040 & -0.167 & 0.027 & 1 & \\
\hline Free AA & 0.167 & 0.212 & 0.188 & 0.142 & 0.130 & -0.110 & 0.162 & $0.703 * *$ & 1 \\
\hline Proline & 0.161 & 0.030 & $0.465^{* *}$ & -0.123 & $0.516^{* *}$ & 0.339 & 0.264 & $-0.363 *$ & -0.209 \\
\hline
\end{tabular}

DM: Dry matter (\%); FW: fresh weight per bulb (g); TS: total soluble sugars (g/100 gm DW); RS: reducing sugars (g/100 gm DW); NRS: non-reducing sugars (g/100 g DW); AIS: alcohol insoluble solids (g/100 g DW); fructans $(\mathrm{g} / 100 \mathrm{~g} \mathrm{DW})$; protein: total soluble protein $(\mathrm{g} / 100 \mathrm{~g} \mathrm{DW})$; free AA: free amino acids (g/100 g DW); proline (g/100 $\mathrm{g}$ DW)

*: Correlation is significant at the $\mathrm{P}<0.05$ level (2-tailed)

**: Correlation is significant at the $\mathrm{P}<0.01$ level (2-tailed)

\section{Conclusions}

The study demonstrated that there are considerable differences for various physico-chemical attributes in red, yellow, and white onion genotypes. Among the three, red genotypes had higher fresh weight, dry weight, total soluble sugars, non-reducing sugars, protein, free amino acids, and proline contents; yellow coloured genotypes showed the highest reducing sugars content, while the maximum AIS content was found in white onion genotypes. Therefore, these coloured genotypes could be exploited for a wide range of uses like fresh 
consumption, processing, and export, and the study would be useful for consumers, farmers, as well as vegetable breeders.

The authors gratefully acknowledge the financial support provided by Punjab Agricultural University, Ludhiana for conducting the experiment.

\section{References}

Armand, A.B., Scher, J., Aboubakar, Augustin, G., Roger, P., ... \& Moses, M.C. (2018): Effect of three drying methods on the physicochemical composition of three varieties of onion (Allium cepa L.). J. Food Sci. Nutr., $1(2), 17-24$.

Azoom, A.A.A., Hamdi, W., Zhani, K. \& Hannachi, C. (2015): Evaluation of mineral element, sugars and proteins compositions in bulbs of eight onion (Allium cepa L.) varieties cultivated in Tunisia. IRJET, 2(4), 35-39.

BATES, L.S. (1973): Rapid determination of free proline content of water-stress studies. Plant Soil, 39, 205-207.

Bhattacharjee, S., Sultana, A., Sazzad, M.H., Islam, M.A., Ahtashom, M.M., Asaduzzaman (2013): Analysis of the proximate composition and energy values of two varieties of onion (Allium cepa L.) bulbs of different origin: A comparative study. IJNFS, 2(5), 246-253.

Dhumal, K., DatiR, S. \& PANDEY, R. (2007): Assessment of bulb pungency level in different Indian cultivars of onion (Allium cepa L.). Food Chem., 100, 1328-1330.

Dubois, M., Gilles, K.A., Hamilton, J.K., Robers, P.A. \& Smith, F. (1956): Colorimetric method for determination of sugars. Anal. Chem., 28, 350-356.

HANCI, F. \& CEBECI, E. (2015): Comparison of salinity and drought stress effects on some morphological and physiological parameters in onion (Allium cepa L.) during early growth phase. Bulg. J. Agric. Sci., 21(6), 1204-1210.

Insani, E.M., Cavagnaro, P.F., Salomón, V.M., Langan, L., Sance, M., ... \& Galmarini, C.R. (2016): Variation for health-enhancing compounds and traits in onion (Allium cepa L.) germplasm. Food Nutr. Sci., 7, 577-591.

Jaime, L., Martin-Cabrejas, M.A., Molla, E., Lopez-Andreu, F.J. \& Esteban, R.M. (2001): Effect of storage on fructan and fructo-oligosaccharides of onion (Allium cepa L.). J. Agr. Food Chem. 49, 982-988.

Jurgiel-Malecka, G., Gibczynska, M. \& Nawrocka-PeziK, M. (2015): Comparison of chemical composition of selected cultivars of white, yellow and red onions. Bulg. J. Agric. Sci. 21(4), 736-741.

Kaur, I., Chawla, N. \& Dhatt, A.S. (2019): Evaluating changes in total soluble sugars, fructans and alcohol insoluble solids in onion (Allium cepa L.) genotypes during ambient storage conditions. Int. J. Chem. Studies, $7(5), 1435-1440$.

KUON, J. \& BERNHARD, R.A. (1963): An examination of the free amino acids of the common onion (Allium cepa). $J$. Food Sci., 28, 298-304.

LeE, Y.P. \& TAKahashi, T. (1966): An improved colorimetric determination of amino acids with the use of ninhydrin. Anal. Biochem., 14, 71-77.

Lowry, O.H., Rosebrough, N.J., Farr, A.L. \& Randall, R.J. (1951): Protein measurement with the folin phenol reagent. J. Biol. Chem., 193, 265-275.

MCRarY, W.L. \& SlatterY, M.C. (1945): The colorimetric determination of fructosan in plant material. J. Biol. Chem., 157, 161.

MOYeR, J.C. \& Holgate, K.C. (1948): Determination of alcohol-insoluble solids and sugar contents of vegetables. Anal. Chem., 20(5), 472-474.

Platel, K. \& SRInivasan, K. (2016): Bioavailability of micronutrients from plant foods: an update. Crit. Rev. Food Sci., 56, 1608-1619.

Rodrigues, A.S., Almeida, D.P.F., Simal-Gándara, J. \& Pérez-Gregorio, M.R. (2017): Onions: A source of flavonoids. -in: Justino, G. (Ed) Flavonoids - from biosynthesis to human health. InTech, Doi:10.5772/ intechopen.69896.

SimON, P.W. (1995): Genetic analysis of pungency and soluble solids in long-storage onions. Euphytica, 82(1), 1-8. Somogyi, M. (1952): Note on sugar determination. J. Biol. Chem., 195(1), 19-23.

Suzuki, M. \& Cutliffe, J.A. (1989): Fructans in onion bulbs in relation to storage life. Can. J. Plant Sci., 69, $1327-1333$.

ViJn, I. \& SMEekens, S. (1999): Fructan: More than a reserve carbohydrate? Plant Physiol., 120, 351-359. 